\title{
Manipulation of Bird Behavior by Parasites?
}

\author{
Anders Pape O-Møller*,1,2
}

${ }^{1}$ Laboratoire d'Ecologie, Systématique et Evolution, CNRS UMR 8079, Université Paris-Sud, Bâtiment 362, F-91405

Orsay Cedex, France ; ${ }^{2}$ Center for Advanced Study, Drammensveien, 78, NO-0271 Oslo, Norway

\begin{abstract}
Many parasites apparently change the behavior of their hosts in a way that seemingly increase the probability of successful reproduction and transmission, suggesting that parasites somehow are able to manipulate the behavior of hosts to their own advantage. Such adaptive manipulation implies that [1] different roles are played by manipulated and manipulator individuals; [2] manipulation reduces the fitness of the manipulated individual; [3] the manipulator gains a fitness advantage; and [4] this order of events should hold up when analyzed in a phylogenetic context. While some examples of parasite-host interactions are consistent with some of these criteria, there is little strict evidence consistent with all four criteria. Parasite manipulation of vertebrate hosts may differ from that of invertebrates because of differences in cognitive ability, and complexity of the parasite community. Literature on avian brood parasites and their hosts suggests that hosts may be fully aware of their parasitism status. Using studies of the great spotted cuckoo and its magpie host I argue that parasitized hosts probably are doing the best they can, given their status, and that their fitness pay-offs would be even worse if they produced higher levels of resistance. Next, I argue that hosts in general may be aware of their infection status, and that each host individual interacts with so many different parasites, each with their 'own' evolutionary interests, that hosts are unlikely to behave only in response to any single parasite. Rather, host behavior could be considered to reflect a compromise between the evolutionary interests of all the inhabitants of a given host individual. Therefore, it might be difficult to argue that hosts are manipulated by parasites, and I suggest that we may learn more about parasitehost interactions by quantifying the evolutionary interests of hosts and their multitude of parasites, amensals and commensals, and that host behavior may more readily be understood from the point of view of the participants involved in these different interspecific interactions.
\end{abstract}

Key Words: Cognition, interspecific interactions, 'mafia' behavior, olfaction, parasite-host interactions, smell.

\section{INTRODUCTION}

Manipulation of host behavior must be clearly defined in order to allow distinction between clear-cut and borderline cases, but also in cases where manipulation is seemingly straightforward clear definitions may allow scientists to pose critical questions. The definition of manipulation assumes that there are individuals manipulated and others manipulating. This also implies that manipulated individuals do not 'behave in their own best interests', but somehow act to the benefits of the manipulator. In other words, the way in which the parasite changes the behavior of the host must have design features that are consistent with the presumed adaptive change in behavior of the host that promotes transmission of the parasite. Such information is far from easy and straightforward to obtain. Take common cold as an example. This condition is caused by rhinovirus infections of the nose and the throat, with sneezing and a runny nose as symptoms. Superficially, sneezing may provide a means for the virus to increase transmission. However, this example may just as well constitute a means for the hosts of ridding themselves of virus. Clearly, we need to quantify the increase in parasite

*Address correspondence to this author at the Laboratoire d'Ecologie, Systématique et Evolution, CNRS UMR 8079, Université Paris-Sud, Bâtiment 362, F-91405 Orsay Cedex, France: Tel: $(+33) 1691556$ 88; Fax: $(+33) 1$ 691556 96; E-mail: anders.moller@u-psud.fr transmission rate attributed to an increase in frequency of sneezing, but we likewise need to quantify the consequences of sneezing for the host in terms of duration of incapacitation or other negative consequences of the infection.

The objectives of this paper are to evaluate our current knowledge of host-parasite interactions, with specific emphasis on parasite manipulation of host behavior in birds. First, I discuss the criteria for identification of parasite manipulation of host behavior. Second, I consider whether we should expect to see parasite manipulation of host behavior in hosts with well-developed cognitive abilities like in birds. Third, I review research on facultative virulence and 'mafialike' behavior by parasites, arguing that tugs of war between parasites and their hosts are widespread, and that their intricacy suggests that hosts may rarely be unaware of their parasite infection status. Fourth, I discuss whether hosts can possibly be unaware of their infection status given the widespread and increasing evidence for avian hosts being able to smell even small differences in parasitic loads. Fifth, I review recent suggestions that any single host individual is infected by many different kinds of parasites, but also by numerous other organisms, each with their own evolutionary interests, suggesting that it is unlikely that any single parasite can successfully manipulate a host entirely to its own advantage. Finally, I conclude by suggesting that we may gain 
more insight into the coevolution of parasite-host interactions and the underlying mechanisms by attempting to view host behavior beyond a single specific interaction.

\section{Sufficient Criteria for Parasite Manipulation of Host Be- havior}

To demonstrate parasite manipulation of host behavior, we will need information on changes in behavior in a phylogenetic context that show that an increment in host behavior from the ancestral state causes an increment in parasite fitness, for example, as shown by increased parasite transmission. Moore and Gotelli [1] have already emphasized the necessity to test this adaptive scenario in a phylogenetic context. Therefore, sufficient and required criteria for demonstration of manipulation include [1] different roles played by manipulated and manipulator individuals; [2] reduction in fitness of the manipulated individual; [3] a gain in fitness by the manipulator; and [4] a specific sequence of events when analyzed in a phylogenetic context. There might be scenarios in which parasites increase their transmission rate without causing a reduction in host fitness, and such scenarios may also qualify as successful manipulation.

The early parasitological literature contains several examples that could be interpreted as evidence of parasite manipulation of host behavior [2]. Prime examples of parasite manipulation of host behavior include the early descriptions of parasites causing hosts to change their behavior in order to facilitate transmission [3]. Likewise, hosts that are castrated by parasites, and in this way increase allocation of resources to parasites [4], may also be considered clear-cut cases of manipulation. The review by Holmes and Bethel [5] provide the first compelling evidence of parasite manipulation of hosts and its underlying assumptions. Several later reviews by Poulin [6-8], including reviews of the underlying mechanisms studied in a phylogenetic perspective [1], provide evidence consistent with the hypothesis of manipulation.

I have summarized the findings by Saumier et al. [15] of Trichinella pseudospiralis infections in the American kestrel (Falco sparverius) as an example of the difficulties encountered when interpreting the evidence for or against parasite manipulation of host behavior. Saunier et al. [15] reported induced behavioral changes within the first 5 days after inoculation that corresponds to the adult phase of the infection. The parasite caused more severe negative effects on mobility of the host when larvae of the parasite migrated and became established in the musculature. This debilitation lasted for at least 5 weeks after inoculation and involved reduced exercising, flying, elevated perching, and preening, and it was accompanied by an increase in frequency of walking and floor perching. Saunier et al. [15] speculated that these behavioral effects, attributable to the presence of larvae in the muscle reduce the competitive ability of infected individuals. The muscle larvae were randomly distributed among various muscle groups. Is this a case of parasite manipulation, and if yes, how would these behavioral changes benefit the transmission of the parasite? Which are the shortcomings of this study? Are there alternative interpretations? Could findings of this study simply reflect pathological effects of the parasite without any real manipulation? I have not singled out the study by Saunier et al. [15] for any particular reason, although I strongly believe that many cases of so-called ma- nipulation have not passed the critical test to assess whether the required criteria for manipulation are fulfilled.

Exceptions to parasite manipulation of host behavior should based on theoretical arguments exist, especially when parasites have complex life cycles [6,9]. Parker et al. [10] have recently investigated in detail the conditions required for parasite manipulation of host behavior. They did so by investigating the evolution of two categories of adaptive host manipulation by trophically transmitted helminths: [1] the parasite reduces the risk of predation of the host before the parasite is ready to become established in a definite host; and [2] the parasite increases the risk of predation when the parasite is ready for transmission to its next host. If all parasite mortality is random, enhancement of host behavior must increase predation by the next host more than it increases the average for all forms of host mortality. In other words, if hosts and parasites only die from randomly acting predation, parasite manipulation of the host must increase predation at the time when the parasite is ready for transmission more than when it is not yet ready. If most parasites die when in their intermediate host before being transmitted, enhancement of predation can evolve if it increases the probability of predation after the parasite is ready for transmission, independent of how much it attracts predators of the 'wrong' species. Enhancement of predation on the host by the parasite is always most favorable when affecting the right host, while suppression of predation promotes survival to the time when establishment in the next host is possible. Suppression of predation is most favorable if it reduces all aspects of host (and hence parasite) mortality. Thus, there are limits to when enhancement of predation can be expected.

Research findings in all fields of research including parasitology are often not based on adequate sample sizes, and experimental designs are often wanting in several respects. Therefore, the magnitude of relationships between any two variables, such as manipulated behavior and parasitism, is a function of the true relationship, but also strongly affected by sample size. At small and even not so small sample sizes, the observed relationship between two variables may for simple sampling reasons be opposite to the 'true' relationship. There are other reasons for expecting strong initial relationships between variables, because a novel hypothesis is unlikely to be published if only supported by a weak empirical basis or no empirical evidence at all. Poulin [11] showed that initially high effect sizes for parasite manipulation of host behavior were followed by decreases, until being indistinguishable from zero. Thus, initially strong effects may have been published only because they were strong. Later, when both 'positive' and 'negative' effects are published, mean effect size levels off at the 'true' level. Jennions and Møller [12] showed that such declines in effect size appear to be ubiquitous in different fields of biology. Initially large effect sizes are often based on small sample sizes that make the estimate of effect size unreliable, contributing to the temporal trend in effect size, associated with an increase in sample size in later published studies [12].

\section{Parasite Manipulation of Vertebrate Hosts: Too Clever by Half?}

A number of different studies have provided evidence consistent with expectations from the manipulation hypothe- 
sis (reviews in $[1,6-8]$ ). There is also evidence suggesting that the behavior of vertebrates may depend on their infection status, including studies of fish [13], reptiles [14], birds [15] and mammals (e. g. [16-19]). There is a huge literature on humans and human parasites, emphasizing how infection may change human behavior in such a way that the parasite or its offspring are released in suitable habitat such as water (e. g. [20-22]). However, we must not forget that for every case of parasite manipulation of the host, there is evolution of host behavior to avoid parasitism or reduce the negative effects of parasitism if infected [23].

Two reasons for emphasizing vertebrates over invertebrates are their larger cognitive abilities and their greater abundance of parasites. The cognitive ability may imply that vertebrates should be more difficult to manipulate if they somehow were able to 'outfox' or discern their manipulators. While vertebrates may have superior cognitive abilities compared to invertebrates, this may both provide advantages and disadvantages. Clearly an advanced brain may provide an individual with cognitive abilities, but simultaneously also constitute a weak spot if it is open to failure when any of its parts are incapacitated. Yet another consequence may be the in-built redundancy of functions and structures in the vertebrate brain as a safeguard against parasite manipulation. This raises the question whether there are specific mechanisms that allow vertebrate hosts to protect their cognitive abilities to be maintained in the face of parasite attack. Comparative analysis of patterns of investment in immunity in birds have suggested that immunity has been shaped to protect brain, learning ability and cognitive ability from debilitating effects of parasites [24]. Whether covariation in brain size and size of immune defense organs of birds, especially in males has evolved as a mechanism to reduce or avoid parasite manipulation remains to be determined, although that is a testable hypothesis.

Cognitive abilities of hosts may be insufficient to prevent host manipulation by parasites, simply because induction of pain, thirst or other responses in the host by the parasite may suffice for enhanced parasite transmission. However, parasite avoidance behavior by hosts, self-medication and numerous other changes in host behavior in response to parasitism (see [24] for an extensive review) clearly demonstrate that hosts are not always readily manipulated.

The second difference for vertebrates is the larger diversity of parasites in hosts with larger body size. Any vertebrate species will probably be the host of hundreds of different parasites, if we assume that parasite diversity in humans and domesticated animals reflect the potential richness of parasites in other species as well. Similarly, Rothschild and Clay [25] show the immense diversity of macro-parasites exploiting the European blackbird Turdus merula, and numerous micro-parasites are also likely to exploit this host. Each of these parasites will attempt to safeguard its specific evolutionary interests. How such diversity in interests is resolved, and which parasite will pull the trigger remains to be determined.

\section{On 'Mafia' Cuckoos, Ignorant Hosts, and Limits to Ad- aptation}

The parasitological literature is based on the notion that parasites cause a reduction in host fitness (i. e., parasitism is costly to hosts), and that this impact of parasite virulence can evolve in response to selection on hosts and parasites (e. $\mathrm{g}$. [26-28]). Often hosts do not suffer from parasitism due to parasites extracting limiting resources from the host, but because parasites inflict damage to the host by tissue destruction, immune responses, and induction of heat shock proteins (e. g. [22,29-30]). This raises the question of what determines the level of damage inflicted by parasites on their host, and whether such damage may change in response to interactions between an individual host and its parasites [31]. Such interactions may lead to facultative virulence that occurs when the parasite changes its behavior in response to the behavior of the host [31]. Any host that complies with parasite demand for limiting resources may suffer less from the negative impact of parasites than a 'resistant' host. Such facultative virulence may be common, as revealed by a number of examples from the literature that are consistent with expectations (a review of such cases can be found in [31]). Facultative virulence may even occur in very 'simple' parasites because the only requirement would be that the parasite has a 'switch' that changes its behavior in response to the state of the host.

The only well documented, albeit not extensively researched example of facultative virulence concerns 'mafia' behavior in brood parasitic cuckoos. The great spotted cuckoo Clamator glandarius is a generalist brood parasite of magpies, starlings and several other large species of birds, laying its eggs in a host nest and being reared together with the offspring of the host that are readily out-competed by the competitively superior parasite [32]. The black-biilled magpie Pica pica is a common host of the great spotted cuckoo in parts of southern Europe. Magpies are well known for their cognitive abilities and their large brain size [33]. Cuckoos impose severe fitness costs on their hosts, reducing reproductive output by half and causing parasitized adults to provide food for cuckoo fledglings for several weeks, thereby considerably delaying the single annual molt. Brood parasites may gain re-nesting opportunities by destroying or preying upon host eggs or nestlings. If hosts change their ejection behavior towards cuckoo eggs in response to parasite destruction of host eggs or nestlings, the parasite will gain a benefit. Such change in host behavior induced by punitive behavior by a parasite is akin to the behavior of mafiosi [34,35]. A host that changed its anti-parasite behavior in response to depredation of the contents of its nest would also benefit because it would be able to raise some offspring of its own, although that number would be reduced compared to the non-existent situation with complete absence of the parasite $[34,35]$. Please note that this is expected only when the parasite chick does not eject all host nestlings. Anecdotal observations of magpies and great spotted cuckoos prompted us to test if the probability of nest predation depended on the fate of the cuckoo egg in a host nest [35]. Magpie nests either had the cuckoo egg removed, or were just visited as a control. The rate of nest predation was increased by a factor of six by the experimental treatment [35]. Surprisingly, destroyed nest contents such as broken eggs and dead nestlings were not eaten, but instead left behind, suggesting that it was not common nest predators like corvids that always eye nest contents that were the perpetrators. Our own observations identified great spotted cuckoos as being responsible for the destruction of host nests, and on a few occasions we could 
even demonstrate that it was the very individual parasite that laid the cuckoo egg that also destroyed the clutch of the host [35]. One possibility is that the parasite may not be 'punishing' the host, but rather just be inducing re-nesting in order to lay a second egg. Induction of re-nesting of the host by destruction of the clutch of the host may be an efficient strategy for the parasite, especially at the beginning of the breeding season. If this was true, then, towards the end of the breeding season, when there is no more time for host renesting, the cuckoo whose egg has been removed should simply eat the remaining host eggs (i. e. it should behave as a predator). We have found no evidence of such predatory behavior by cuckoos. We can distinguish between 'punishment' and induction of re-nesting as a cause of destruction of host clutches by the actual behavior of the host. If the host ejected the cuckoo egg, destruction of the clutch of the host was much more likely than if the host accepted, independent of the timing of parasitism relative to laying by the host [35].

To illustrate the complexities of these interactions between parasites and hosts, I will briefly present an eyewitness report. Together with Juan J. Soler I was doing fieldwork in Guadix, Southern Spain, in early April 1995. We were checking magpie nests for egg laying and parasitism. Juan had just checked a magpie nest in an almond tree, and it contained five eggs, but the clutch was not yet finished. There were no magpies around, and when Juan returned from the nest and was writing notes, a great spotted cuckoo arrived and flew directly into the nest. A few second later it exited the magpie nest, and I suggested to Juan that we check the nest again. Sure enough, the nest now contained a cuckoo egg and five magpie eggs, and all eggs were undamaged. I suggested that Juan removed the cuckoo egg to see what would happen. Juan returned to ground level once more, this time with the cuckoo egg, while the cuckoo was sitting in a nearby tree. Then it flew directly back to the magpie nest, stayed there for a few second, and exited again. We had no choice than to check the nest once more! There were still five magpie eggs in the nest, but all of them had peck marks and were rendered unviable by the cuckoo. The cuckoo was still sitting in the nearby tree, but eventually flew away. An alternative interpretation of these events is that the cuckoo simply perceived the presence of a human as a potential risk of predation. Why the behavior of the cuckoo in this case should involve destruction of the clutch of the host in response to its own egg missing cannot readily be explained. If anything, a cuckoo that guarded a host nest against predation by a potential predator would be expected to give alarm calls and even actively defend the host nest from the very beginning. That was not the behavior performed by the cuckoo. What this observation could tell us was that the cuckoo responded to the absence of its egg (or an egg, alternatively), and that it responded to this absence by destroying the eggs of the host without eating them. Clearly, it was not required that the magpie was present for eliciting this behavior by the cuckoo, and we suggest that it was the absence of the cuckoo egg that caused this change in parasite behavior.

Having obtained empirical evidence consistent with the idea that cuckoos acted as 'mafiosi', providing 'an offer that hosts could not refuse', we conducted a second experiment in two magpie populations, one heavily parasitized by great spotted cuckoos, and one rarely parasitized. This time we imtended to test if the 'mafia-like' behavior of the parasite caused a change in the behavior of the host [36]. We created three experimental groups of which two were tested for rejection behavior of artificial cuckoo eggs. In the first of these two groups both ejecters and accepters had their nests depredated by the experimenter, and ejection behavior of pairs that re-laid was subsequently tested. In the second of the two groups we only tested for ejection. The third group that constituted a control group was not subject to recognition tests, but was experimentally depredated, followed by ejection tests. This latter group in other words constituted a control group to test for effects of the ejection test on later ejection behavior. In the heavily parasitized population there was a significant reduction in ejection behavior by magpies in experimental nests that were experimentally depredated, but not in control nests. In the weakly parasitized population there was no significant change in ejection behavior in either group. Thus, this experiment demonstrated that magpies changed their anti-parasite behavior in response to experimental destruction of the contents of their nests, but only in heavily parasitized host populations where there was a high probability to parasitism even in replacement clutches. We found a certain level of nest predation in the control group (with the typical signs of predation: broken egg shells and nest contents missing), and we assume that a similar level of predation occurred in experimental nests (see also [35] for similar data on differences in destruction of nest contents by nest predators and cuckoos). Recent research on ejection behavior of the magpie host has shown that ejection of cuckoo eggs (or model eggs) to a large extent is determined by a quantitative trait locus (QTL) that is closely linked to a specific microsatellite [37]. Subsequent research has revealed that spatial variation in the frequency of alleles of the QTL provide information on spatial variation in ejection behavior [38]. These results suggest that ejection behavior is partly under genetic control, but also that residual variation exists, allowing for phenotypic plasticity that could be exploited by a mafioso-like cuckoo. Clearly, it would now be interesting to repeat some of the previous 'mafia' experiments to test if a 'mafia-like' cuckoo differentially depredates nests of hosts with accepter alleles.

\section{Do Hosts Know That they are Parasitized? Smelly Para- sites and Host Olfaction}

Do hosts know that they are parasitized? Brood parasites constitute odd examples of parasites because hosts that are often the size of a fraction of the parasitic cuckoo continue to feed the cuckoo chick well beyond the normal nestling period. Given that humans readily can tell the difference between the parent host and the parasitic cuckoo, this raises the question why the host cannot, and why it continues to feed the parasite. Recent research on two different species of cuckoos suggests that hosts of brood parasites indeed are able to tell the difference. For example, Grim et al. [39] showed for reed warblers Acrocephalus scirpaceus that parasitized individuals incubated the cuckoo egg and its own eggs, watched the cuckoo nestling eject its own eggs, and fed the cuckoo chick well beyond the normal size of reed warbler young. Almost all studies of cuckoos have only lasted 
until the start of incubation, or the beginning of the nestling period. In contrast, Grim et al. [39] followed the cuckoo nestling until after fledging, showing that many of these fledglings were abandoned by hosts and therefore subsequently died. This constitutes clear evidence of cuckoo hosts knowing their status of parasitism and behaving accordingly. Langmore et al. [40] provided similar evidence for a native cuckoo from Australia. We could question whether these are cases of hosts being 'aware' of their parasite status, or of host parents reaching the limits of their parental care beyond which their residual reproductive value would be jeopardized. Because cuckoo hosts may still have time for re-laying after abandoning a fledged cuckoo [41], I consider it unlikely that cuckoo hosts are unaware of their status of parasitism. Cuckoos do generally not parasitize hosts with fledglings that reach independence from parental care at an age commensurate with cuckoo development. These studies suggest that we should be careful to make claims about hosts being unaware of their status of parasitism based on limited evidence.

Birds have recently been shown to have an excellent sense of smell and use this to their own advantage [42]. For example, Amo et al. [43] have shown for blue tits Cyanistes caeruleus that individuals change their behavior in response to experimental application of the smell of a potential predator to the nest. Are we to believe that cuckoos and other brood parasites would be less smelly than predators that just visit a nest for a very short time? Is it possible that the rapid pace of egg laying by brood parasites is in fact an adaptation to avoid deposition of smell in the nest of the host? These speculations, albeit likely speculations, raise the question whether cuckoo hosts and hosts of other brood parasites really are manipulated. Alternatively, we might expect that such hosts in fact are doing the best they can, given their options.

The significance of smell as a consequence of parasitism may also apply to other host-parasite systems because parasitism often changes the smell of hosts. Many human infectious diseases are accompanied by characteristic smells that can readily be identified by other humans, but also by the infected individual (e. g. $[44,45])$. Similar information exists for mice [46]. This raises questions about the ability of individuals to know their current infection status and to change their behavior accordingly.

\section{Is it Likely That Parasite Manipulation of Hosts Should Succeed in the Face of the Evolutionary Interests of Other Players?}

No host is an island, and no parasite is alone on a host. This almost trivial statement leaves the open question whether we should expect parasite manipulation of hosts to be common, or even discernible. While successful manipulation of hosts would suggest that a parasite somehow managed to change the behavior of the host to its own advantage, and hence 'over-rule' all other co-inhabitants of this single host individual that might not benefit from host manipulation, we might still question whether and when this is ever likely. For example, parasite-host interactions may affect the probability of predation [47]. Whether birds are infected with blood parasites is an important determinant of suscepti- bility to generalist Accipiter hawks [48], and the probability that a parasite infecting an intermediate host may end up in a final host will strongly depend on the community of predators and their prey preferences. To complicate matters further, predators may depress the ability of hosts to produce immune responses, thereby increasing the prevalence and the intensity of infection with blood parasites [49]. Furthermore, immune factors of hosts exposed to predators may be transmitted to the next generation as maternal effects [50]. Thus, host-parasite interactions do not exist in a vacuum and they are likely to interact with other interactions [51]. The interaction between a parasite and its host will be affected by interactions between the host and potential predators, but also numerous other inter-actors [51]. For example, the number of different microorganisms inhabiting an average human amounts to several hundred 'species' and their combined weight is several kilograms [52]. Each of these players will be positively or negatively affected by the manipulation by a single parasite, and they are all expected to act in their own best evolutionary interest. This point of view is also consistent with one theory about the evolution of the immune system, suggesting that immunity has just as much evolved to facilitate the benefits of symbionts and commensal organisms as the fight against parasites [53]. These reflections clearly lead to the question about who is in charge of the behavior of a host? Is it the host itself that affects its behavior, is it a 'virulent' parasite, is it the community of commensals, or is it a combination of all these different players relative to their share of the 'power' over the future of the host individual? Clearly, these are difficult questions to answer, and I do not think there are any simple answers. However, they do leave the impression that it would be highly unlikely that a single or a few players would be 'able to pull it off' repeatedly to their own advantage on an evolutionary time scale that would be required for adaptations to evolve. This brings home the point that perhaps we should not expect such manipulation to be commonplace.

\section{Where Does Parasite Manipulation of Hosts Lead? Or, Where We go from Here?}

In this paper I have tried to argue that we often do not know anything about how individuals perceive their own infection status, that individuals typically are infected by numerous species of parasites, amensals and commensals, and that each of these organisms have its own evolutionary interests. Because parasite-host interactions also depend on other interspecific interactions such as predator-prey interactions and mutualistic interactions between symbionts and their hosts, it seems unlikely that any single inhabitant of a host will ever be able to manipulate host behavior to its own exclusive advantage. Rather, it seems likely that host behavior can be understood as a consequence of the different evolutionary interests of a host individual and all its coinhabitants, given the environment in which that individual is living. We may make progress in understanding the evolution of parasite-host interactions and the underlying mechanisms by trying to view host behavior beyond a specific interaction. The evolution of virulence and the fitness costs that parasites inflict on their hosts may also be better understood if the interests of the different interacting parties that include host, parasites and various amensals and commensals 
are fully considered. Who is going to present the first model of evolution of virulence in the simultaneous presence of mutualists that may try to 'rescue' a host?

\section{ACKNOWLEDGEMENT}

A. Poiani kindly invited me to contribute this paper and provided constructive criticism. An anonymous reviewer helped clarify my points of view.

\section{REFERENCES}

[1] Moore J, Gotelli NJ. In: Barnard CJ, Behnke JM, Eds. Parasitism and host behaviour, London: Taylor \& Francis 1990; 193-233.

[2] Wesenberg-Lund C. Contributions to the development of the trematoda digenea. I. the biology of leucochloridium paradoxum. Mém Acad R Sci Lett Danemark Sect Sci 1931; 4: 90-142.

[3] Hindsbo O. Effects of polymorphus (acanthocephala) on the colour and behaviour of gammarus lacustris. Nature 1972; 238: 333.

[4] Kuris AM. Trophic interactions: similarity of parasitic castrators to parasitoids. Q Rev Biol 1974; 49: 129-48.

[5] Holmes JC, Bethel WM. In: Canning EU, Wright CA, Eds. Behavioural aspects of parasite transmission London: Academic Press 1972; 123-49.

[6] Poulin R. The evolution of parasite manipulation of host behaviour: a theoretical analysis. Parasitology 1994a; 109: S109-18.

[7] Poulin R. Meta-analysis of parasite-induced behavioural changes. Anim Behav 1994b; 48: 137-45.

[8] Poulin R. Adaptive changes in the behaviour of parasitized animals: a critical review. Int J Parasitol 1995; 25: 1371-83.

[9] Brown SP. Cooperation and conflict in host-manipulating parasites. Proc R Soc Lond B 1999; 266: 1899-904.

[10] Parker GA, Ball MA, Chubb JC, Hammerschmidt K, Milinski M. When should a trophically transmitted parasite manipulate its host? Evolution 2009; 63: 448-58

[11] Poulin R. Manipulating host behaviour by parasites: a weakening paradigm? Proc R Soc Lond B 2000; 267: 787-92.

[12] Jennions MD, Møller AP. Relationships fade with time: a metaanalysis of temporal trends in publication in ecology and evolution. Proc R Soc Lond B 2002; 269: 43-8.

[13] Krause J, Godin J-G J. Influence of parasitism on the shoaling behaviour of banded killifish, fundulus disphanus. Can J Zool 1994; 72: 1775-9.

[14] Daniels CB. The effect of infection by a parasitic worm on swimming and diving by the water skink, Sphenomorphus quoyii. J Herpetol 1985; 19: 160-2.

[15] Saumier MD, Rau ME, Bird DM. The influence of trichinella pseudospiralis infection on the behaviour of captive, nonbreeding american kestrels (falco sparverius). Can J Zool 1988; 66: 1685-92.

[16] Rau ME. The open-field behaviour of mice infected with trichinella spiralis. Parasitology 1983; 86: 311-8.

[17] Hay J, Aitken PP. Experimental toxocaris in mice and its effect on their behaviour. Ann Trop Med Parasitol 1984; 78: 145-55.

[18] Webster JP. The effect of toxoplasma gondii and other parasites on activity levels in wild and hybrid rattus norvegicus. Parasitology 1994; 109: 583-9.

[19] Cox DM, Holland CV. The relationship between numbers of larvae recovered from the brain of toxocara canis-infected mice and social behaviour and anxiety of the host. Parasitology 1998; 116: 579-94.

[20] Beaver PC, Jung RC, Eds. Animal agents and vectors of human disease. Philadelphia: Lea \& Febiger 1985.

[21] Beck JW, Davies JE. Medical parasitology, $3^{\text {rd }}$ ed. St. Louis: CV. Mosby; 1981.

[22] Conner DH, Chandler FW, Schwartz DA, Manz HJ, Lack EE, Eds. Pathology of infectious disease. Stamford: Appleton \& Lange 1997.

[23] Hart BJ. Behavioral adaptations to pathogens and parasites: five strategies. Neurosci Biobehav Rev 1990; 14: 273-94.

[24] Møller AP, Erritzøe J, Garamszegi LZ. Coevolution between brain size and immunity in birds: implications for brain size evolution. $\mathbf{J}$ Evol Biol 2005; 18: 223-7.
Rotschild M, Clay T. Fleas, flukes and cuckoos. London: Collins; 1952.

[26] Bull JJ. Virulence. Evolution 1994; 48: 1423-37.

[27] Ewald P. The evolution of infectious diseases. Oxford: Oxford University Press; 1994.

[28] Frank SA. Models of parasite virulence. Q Rev Biol 1996; 71: 37 78 .

[29] Merino S, Martínez J, Møller AP, Barbosa A, de Lope F, Rodríguez-Caabeiro F. Blood stress protein levels in relation to sex and parasitism of barn swallows (Hirundo rustica). Ecoscience 2002; 9: 300-05.

[30] Wakelin D. Immunity to parasites. $2^{\text {nd }}$ ed. Cambridge: Cambridge University Press 1996

[31] Soler JJ, Møller AP, Soler M. Mafia behaviour and the evolution of facultative virulence. J Theor Biol 1998; 191: 267-77.

[32] Soler JJ, Soler M. Brood-parasite interactions between great spotted cuckoos and magpies: a model system for studying coevolutionary relationships. Oecologia 2000; 125: 309-20.

[33] Birkhead TR. The magpies. London: T \& A D Poyser; 1991.

[34] Møller AP, Soler JJ, Soler M, Martínez JG. Mafia great spotted cuckoos and magpie host behaviour. Ric Biol Selvaggina 1995; 22: 61-6.

[35] Soler M, Soler JJ, Martinez JG, Møller AP. Magpie host manipulation by great spotted cuckoos: evidence for an avian mafia? Evolution 1995; 49: 770-5.

[36] Soler JJ, Sorci G, Soler M, Møller AP. Change in host rejection behavior mediated by the predatory behavior of its brood parasite. Behav Ecol 1999; 10: 275-80.

[37] Martín-Gálvez D, Soler JJ, Martínez JG, Krupa AP, Richard M, Soler M, Møller AP, Burke T. Genetic basis for recognition of foreign eggs in the host of a brood parasite. J Evol Biol 2006; 19: 54350 .

[38] Martín-Galvez D, Soler, JJ, Martínez JG, Krupa AP, Soler M, Burke T. Cuckoo parasitism and productivity in different magpie subpopulations predict frequencies of the $457 \mathrm{bp}$ allele: a mosaic of coevolution at a small geographic scale. Evolution 2007; 61: 23408.

[39] Grim T, Kleven O, Mikulica O. Nestling discrimination without recognition: a possible defence mechanism for hosts towards cuckoo parasitism. Proc R Soc Lond B 2003; 270: S73-5.

[40] Langmore N, Hunt S, Kilner RM. Escalation of a coevolutionary arms race through host rejection of brood parasitic young. Nature 2003; 422: 157-60.

[41] Pagel M, Møller AP, Pomiankowski A. Retaliatory cuckoos select for hosts that rear cuckoo nestlings. Behav Ecol 1998; 9: 566-72.

[42] Hagelin JC. Odors and chemical signaling. In: Reproductive behavior and phylogeny in birds. BMG Jamieson. Enfield: Science Publishers 2007; vol. 6B: pp. 76-119.

[43] Amo L, Galván I, Tomás G, Sanz JJ. Predator odour recognition and avoidance in a songbird. Funct Ecol 2008; 22: 289-93.

[44] Liddell K. Smell as a diagnostic marker. Postgrad Med J 1976; 52 : 136-8.

[45] Pavlou AK, Turner AP. Sniffing out the truth: clinical diagnosis using the electronic nose. Clin Chem Lab Med 2000; 38: 99-112.

[46] Kavaliers M, Choleris E, Pfaff DW. Genes, odours and the recognition of parasitized individuals by rodents. Trends Parasitol 2005; 21: 423-9.

[47] Temple SA. Do predators always capture substandard individuals disproportionately from prey populations? Ecology 1986; 68: 66974.

[48] Møller AP, Nielsen JT. Malaria and risk of predation: a comparative study of birds. Ecology 2007; 88: 871-81.

[49] Navarro C, Møller AP, Marzal A, de Lope F. Predation risk, host immune response and parasitism. Behav Ecol 2004; 15: 629-35.

[50] Saino N, Romano M, Ferrari RP, Møller AP. Stressed mothers produce low-quality offspring with poor fitness. J Exp Zool 2005; 303A: 998-1006.

[51] Møller AP. Interactions between interactions: predator-prey, parasite-host and mutualistic interactions. NY Acad Sci 2008; 1133: 180-6. 
[52] Turnbaugh PJ, Ley RE, Hamady M, Fraser-Liggett CM, Knight R, Gordon JI. The human microbiome project. Nature 2007; 449: 80410
[53]

Travis J. On the origin of the immune system. Science 2009; 324: $580-2$

Received: April 24, 2009

Revised: May 04, 2009

Accepted: May 05, 2009

(c) Anders Pape Moller; Licensee Bentham Open.

This is an open access article licensed under the terms of the Creative Commons Attribution Non-Commercial License (http://creativecommons.org/licenses/_by-nc/3.0/) which permits unrestricted, non-commercial use, distribution and reproduction in any medium, provided the work is properly cited. 